\title{
Validation Study of GENE on ASDEX Upgrade Using Perturbative Thermal Diffusivity Measured with Partial Sawtooth Heat Pulses
}

\author{
A.J. Creely ${ }^{1}$, T. Görler ${ }^{2}$, G.D. Conway ${ }^{2}$, S.J. Freethy ${ }^{1,2}$, \\ N.T. Howard ${ }^{1}$, P.A. Schneider ${ }^{2}$, A.E. White ${ }^{1}$, M. \\ Willensdorfer ${ }^{2}$, and the ASDEX Upgrade Team \\ ${ }^{1}$ MIT Plasma Science and Fusion Center, Cambridge, 02139 MA, USA. \\ 2 Max Planck Institute for Plasma Physics, 85748, Garching, Germany.
}

\begin{abstract}
.
Perturbative thermal diffusivity is measured with partial sawtooth-generated heat pulses on ASDEX Upgrade for the first time, and these measurements are used to validate the first nonlinear ion-scale gyrokinetic simulation that agrees with experimentally measured perturbative diffusivity. Recent work on Alcator C-Mod [N.T. Howard et al., Phys. Plasmas 23, 056109 (2016)] and DIII-D [C. Holland et al., Nucl. Fusion 57, 066043 (2017)] has shown that ion-scale gyrokinetics cannot adequately describe certain plasma conditions, and that in these instances multi-scale simulations may resolve the observed discrepancies. This work presents the first measurements of electron perturbative thermal diffusivity with partial sawteeth on ASDEX Upgrade (measuring values between 2 and $9 \mathrm{~m}^{2} / \mathrm{s}$ ), and compares these measurements to those made with more established modulated electron cyclotron heating measurements, finding good agreement within experimental uncertainty. Perturbative diffusivity is found to scale inversely with collisionality across ASDEX Upgrade and Alcator C-Mod plasmas. Finally, perturbative diffusivity is used as a validation constraint in a study with the gyrokinetic code GENE, showing the first instance where an ion-scale gyrokinetic simulation can simultaneously match the experimental ion and electron heat fluxes and the perturbative thermal diffusivity. These results indicate that multi-scale effects may only be important in some plasmas, and the collisionality and ratio of high to low wavenumber linear growth rates may distinguish these cases.
\end{abstract}

PACS numbers: 52.55.Fa

Keywords: Perturbative Transport, Thermal Diffusivity, Sawtooth

Submitted to: Nucl. Fusion 


\section{Introduction}

As theoretical models of turbulent transport in fusion plasmas become ever more developed, it is increasingly important to rigorously compare these models to experiment to ensure that they are faithfully reproducing experimental observations. Only then can these codes be used in good faith for the prediction of future machines or of future plasmas in current machines. Validation, or the process of determining how accurately a model represents reality insofar as is needed for the intended purpose of the model [1], has therefore become a key element in the evolution of gyrokinetic and other turbulent transport models.

Validation of gyrokinetic codes, such as GENE 2, has traditionally involved comparison of the predicted and experimental ion and electron heat fluxes, as these would appear to be the most relevant if the ultimate goal is the prediction of future machine performance. More recent work, however, has revealed that such comparisons are susceptible to fortuitous agreement, and that additional comparisons (fluctuation levels, etc.) are required in order to truly assess the accuracy of these codes [3, 4]. In such comparisons, experimental quantities that can be more directly measured are typically given more weight than those that must be inferred. In addition to experimentally measured temperature and density fluctuation levels, the electron perturbative thermal diffusivity, $\chi_{e}^{\text {pert }}$, has also recently been used to further constrain gyrokinetic simulations as part of validation studies [5, 6, 7, 8].

The perturbative thermal diffusivity differs from the power balance diffusivity, $\chi_{e}^{P B}$, in both its definition and interpretation [9, [10]. The power balance thermal diffusivity is defined as:

$\chi_{e}^{P B}=\frac{Q_{e}}{n_{e} \nabla T_{e}}$

where $Q_{e}$ is the electron heat flux, $\nabla T_{e}$ is the radial temperature gradient, and $n_{e}$ is the electron density 9. This quantity has units of $\mathrm{m}^{2} / \mathrm{s}$ and governs steady state transport of heat through the plasma.

On the other hand, the perturbative (or incremental) thermal diffusivity is defined as:

$\chi_{e}^{p e r t}=\frac{1}{n_{e}} \frac{\partial Q_{e}}{\partial \nabla T_{e}}$

This quantity governs the propagation of heat pulses through the plasma and is related to the profile stiffness in the plasma, where stiffness is the incremental change in flux for an incremental change in gradient above the critical gradient [11. The perturbative thermal diffusivity also has units of $\mathrm{m}^{2} / \mathrm{s}$.

In particular, perturbative diffusivity is of interest given recent work with multi-scale gyrokinetic simulations, which indicates that in some plasma conditions, ion-scale simulations cannot adequately match experimental heat fluxes and perturbative diffusivity, and that multi-scale effects are required to resolve these discrepancies 6, 8, 12, 13.

In addition to the motivation of validating transport models, studying experimental trends in the value of the perturbative diffusivity offers insight into the manner in which the plasma response to changing gradients depends on other plasma parameters. These parametric trends may help further characterize the nature of turbulence experimentally, leading to new theoretical insights. One example of the dependence of the perturbative diffusivity on various plasma parameters in Alcator C-Mod can be found in Reference 5.

This paper presents the first measurements of the perturbative thermal diffusivity on ASDEX Upgrade using the propagation of partial sawteeth-generated heat pulses, and uses these measurements for the first time in a validation study of the gyrokinetic code GENE. Section 2 describes the method of measuring the perturbative thermal diffusivity, originally used on Alcator C-Mod 5, and compares two different specific calculation procedures. Section 2 also presents the first comparison of perturbative diffusivity measurements made with the partial sawteeth method to measurements made with the more established modulated electron cyclotron heating method, finding good agreement within experimental uncertainty. Section 3 then presents experimental observations of how the measured perturbative diffusivity scales with plasma collisionality, including a cross-machine comparison of both Alcator C-Mod and ASDEX Upgrade data. Finally, Section 4 utilizes perturbative diffusivity measurements from one discharge, as well as power balance modeling, to validate local, ion-scale, nonlinear GENE simulations.

\section{Perturbative Diffusivity Analysis Method}

The perturbative thermal diffusivity measurements in this paper are based on the tracking of heat 
pulses generated by partial sawtooth crashes, a method originally described in Reference [5]. As the heat pulse propagates outward, its position and amplitude are measured with various channels, spaced by approximately $1.7 \mathrm{~cm}$, of a profile electron cyclotron emission (ECE) radiometer [14 on ASDEX Upgrade. Using partial sawteeth, rather than full sawteeth, to generate the heat pulses avoids complications due to the presence of the 'ballistic effect' in full sawteethgenerated heat pulses [15, 16]. Partial sawtooth crashes occur in an annular region of the plasma, such that the temperature drops significantly slightly offaxis but remains steady on-axis. The perturbative thermal diffusivity, $\chi_{e}^{\text {pert }}$, can then be calculated via the "Extended-Time-to-Peak" method [17, 18, which relates the perturbative thermal diffusivity to the velocity of the peak of the heat pulse and the decrease in the amplitude of the heat pulse as it propagates radially outward. This method inherently gives a radially averaged measurement, over the radial range through which the pulse propagation is tracked.

Note that it would also be useful to measure the ion perturbative thermal diffusivity, but such measurements are often very difficult, considering that in many plasmas the ion heat pulses are of smaller amplitude and that often ion temperature measurements have larger diagnostic uncertainty. See Reference 19] for one example of such measurements.

This section will first compare two specific implementations of this calculation, one that calculates the perturbative diffusivity for each individual heat pulse, and one that utilizes many pulses to form a single 'composite' heat pulse, for which the perturbative diffusivity is then calculated. The section will then present the first comparison of measurements made by the partial sawtooth based method of measuring the perturbative diffusivity with the more established modulated electron cyclotron heating $(\mathrm{ECH})$ based method [9, 20, 21, 22, 23, 24]. The two methods will be shown to agree within experimental uncertainty.

\subsection{Composite Sawtooth Comparison}

One of the differences between the implementation of the Extended-Time-to-Peak method in Reference [5] and in previous work (for example, Reference [17]) is that Reference [5] calculated $\chi_{e}^{\text {pert }}$ for each partial sawtooth, and then averaged the values together to obtain a mean value for the plasma discharge, while previous work has typically created an average sawtooth by superimposing many crashes before the calculation. While these two methods are expected to produce the same answer to within experimental uncertainty, such a comparison had not been performed before this study. As part of commissioning the method on ASDEX Upgrade, this study compared
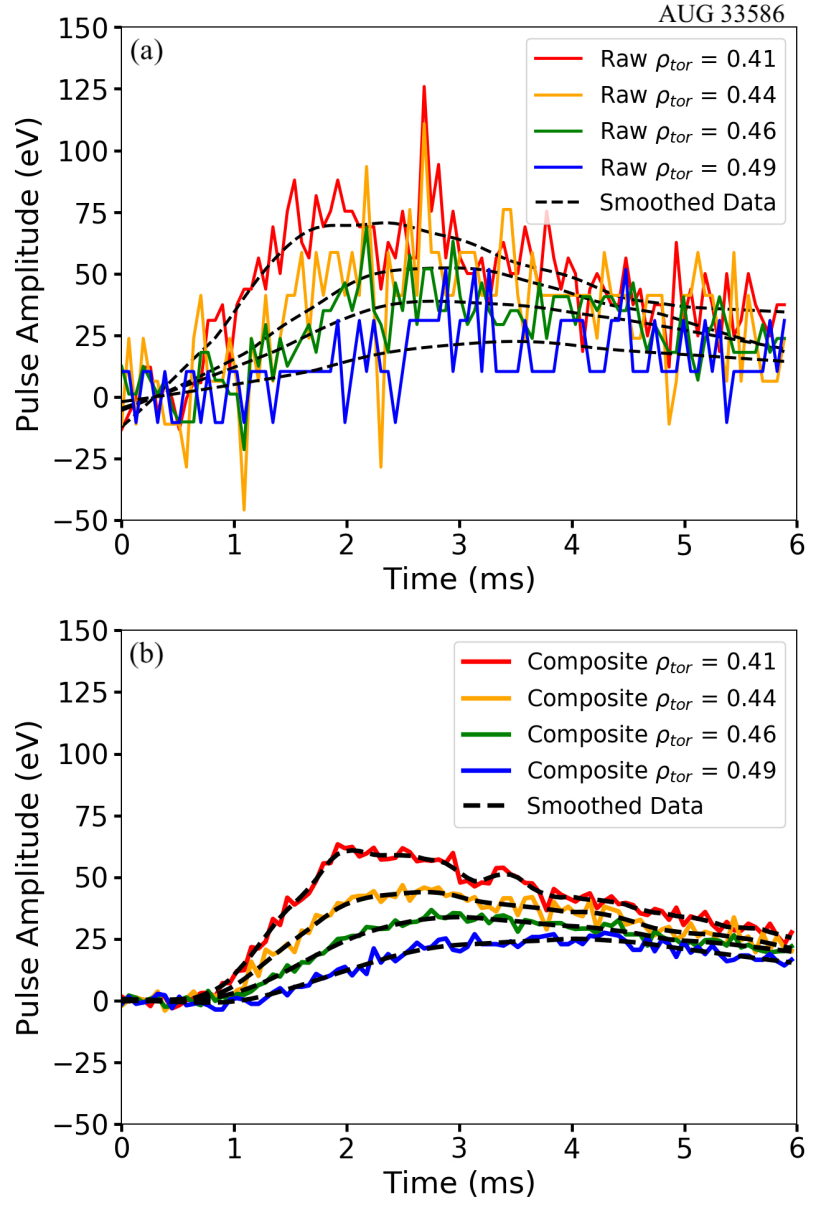

Figure 1: Example measurements of partial sawtoothgenerated heat pulses from ASDEX Upgrade discharge 33586. (a) shows a single pulse propagating through four measurement channels, along with the smoothed data. (b) shows a composite pulse formed by combining data from 36 individual pulses, including the data shown in (a). $\rho_{\text {tor }}$ is the square root of the normalized toroidal flux.

calculations from many individual partial sawtooth heat pulses and from a single composite partial sawtooth heat pulse.

Figure 1 shows an individual heat pulse and a composite heat pulse on ASDEX Upgrade (discharge \#33586) generated by partial sawtooth crashes. The composite heat pulse was generated by averaging 36 individual heat pulses together, including the individual pulse shown. The peak amplitude of the innermost radial (and thus largest amplitude) channel was utilized to synchronize the heat pulse signals for averaging. Both of these traces also show smoothed data, smoothed using a lowess (locally weighted scatterplot smoothing) algorithm, which is ultimately used to calculate the perturbative thermal diffusivity. The smoothing process is very similar to 
that originally used in Reference [5].

As seen in Figure 1. the composite sawtooth visually shows the propagation of the heat pulse much more cleanly. Numerically, however, the difference between averaging $\chi_{e}^{\text {pert }}$ calculated from many individual heat pulses and calculating $\chi_{e}^{\text {pert }}$ from a single composite heat pulse falls within experimental uncertainty. In the particular discharge shown in Figure 1, averaging 36 individual heat pulse calculations gives a result of $\chi_{e}^{\text {pert }}=5.0 \pm 1.4 \mathrm{~m}^{2} / \mathrm{s}$ (with the uncertainty calculated from a combination of diagnostic uncertainty and the standard deviation of the calculated values [5]), while calculating $\chi_{e}^{\text {pert }}$ based on a composite of the exact same set of heat pulses results in $\chi_{e}^{\text {pert }}=4.2 \mathrm{~m}^{2} / \mathrm{s}$. These two values agree within experimental uncertainty, and some of the discrepancy may be attributed to the chosen method of synchronizing pulses in the composite.

The agreement between these two methods of calculating the perturbative diffusivity is robust across many discharges. Using a larger set of data, 24 plasma discharges on ASDEX Upgrade, the composite heat pulse method and the averaged individual heat pulse method agree within one standard deviation $67 \%$ of the time (16 of 24 discharges), which is entirely consistent with a normal distribution of error. This result shows that these two methods of calculation are indeed equivalent, and can be used interchangeably in future work.

\subsection{Modulated ECH Comparison}

In addition to confirming that calculating $\chi_{e}^{\text {pert }}$ from averaging individual heat pulses and from a composite heat pulse generates the same result, this study also compares $\chi_{e}^{\text {pert }}$ measured via partial sawteeth to $\chi_{e}^{\text {pert }}$ measured with modulated Electron Cyclotron Heating $(\mathrm{ECH})$. This is perhaps the more important comparison, as it confirms that the partial sawtooth method, which is still relatively novel, agrees with the more widely accepted modulated ECH method of measuring $\chi_{e}^{\text {pert }}[9,20,21,22,23$. Since the partial sawtooth method is entirely passive and can measure the perturbative diffusivity in many plasmas without the need for dedicated experiments or hardware, there is considerable motivation to confirm that this new method agrees with modulated ECH measurements. The comparison made in this study shows that the modulated ECH and partial sawtooth heat pulse methods agree to within experimental uncertainty in the cases analyzed here.

The modulated ECH measurements that will be shown here are originally described in Reference [25. Modulated ECH and partial sawtooth measurements of $\chi_{e}^{\text {pert }}$ were both available in discharge \#31369, and so the two measurements are compared from the same discharge. The other discharge described in Reference [25], \#30693, did not have a sufficient number of partial sawtooth crashes to make a reasonable measurement via partial sawtooth heat pulses. This discharge was, however, repeated immediately afterward, and this repeat, 30694, did have enough partial sawtooth heat pulses to measure via this method. For this reason, one of the modulated ECH and partial sawtooth comparisons will be made for the same discharge, \#31369, while the other will be made between two repeat discharges, \#30693 and \#30694. Both sets of discharges were $0.8 \mathrm{MA}, 2.5 \mathrm{~T}, 4.0 \cdot 10^{19} \mathrm{~m}^{-3}$ lineaveraged density, electron-heated L-modes.

The modulated ECH method of measuring perturbative thermal diffusivity involves generating heat pulses in the plasma by modulating electron cyclotron heating on and off at a relatively high frequency $(100 \mathrm{~Hz}$ in the plasmas shown here) for long enough to obtain good statistics on the pulse propagation 9, 20, 21, 22, 23. The perturbative thermal diffusivity is then calculated using a method based on Fourier transforms of the data, originally presented in Reference [26]. The Fourier and Extended-Time-to-Peak methods have been shown to agree to within experimental uncertainty (approximately 20\%) 27]. Please see Reference 25] for further details of the analysis utilized on the discharges shown here.

Table 1 shows the results of both partial sawtooth and modulated ECH measurements of $\chi_{e}^{\text {pert }}$ for discharges \#31369 and \#30693/4. One must note that while the two measurements were made in very similar radial locations, they do not quite overlap. The modulated ECH $\chi_{e}^{\text {pert }}$ was measured at $\rho_{\text {tor }} \approx$ $0.34-0.44$, while the partial sawtooth measured at $\rho_{\text {tor }} \approx 0.23-0.29$. The partial sawtooth measurement was constrained by the sawtooth mixing radius and the radius beyond which the heat pulses were no longer detectable. The modulated ECH was constrained by ECH deposition location and the region where the sawtooth interference is too large [25].

With the caveat that the two measurement locations don't quite overlap, measurements with modulated ECH and with partial sawteeth agree to within experimental uncertainty in both cases. While the perturbative thermal diffusivity will likely vary with radius, the gap between two measurement locations is small enough (approximately $3 \mathrm{~cm}$ ) that comparing the two measurements directly is still worthwhile. In both cases the measurements agree within uncertainty, successfully verifying the partial sawtooth method of measuring $\chi_{e}^{\text {pert }}$ against the more widely accepted modulated ECH method.

Now that the method of measuring perturbative thermal diffusivity has successfully been applied to ASDEX Upgrade discharges and has been checked 


\begin{tabular}{|c|c|c|c|c|}
\hline & \multicolumn{2}{|c|}{ Modulated ECH } & \multicolumn{2}{c|}{ Partial Sawteeth } \\
\hline Shot & $\begin{array}{c}\text { Radial Range } \\
\left(\rho_{\text {tor }}\right)\end{array}$ & $\chi_{E C H}^{\text {pert }}\left(\mathrm{m}^{2} / \mathrm{s}\right)$ & $\begin{array}{c}\text { Radial Range } \\
\left(\rho_{\text {tor }}\right)\end{array}$ & $\chi_{P S T}^{\text {pert }}\left(\mathrm{m}^{2} / \mathrm{s}\right)$ \\
\hline $30693 / 4$ & $0.34-0.44$ & $2.2 \pm 0.3$ & $0.22-0.29$ & $2.1 \pm 0.6$ \\
\hline 31369 & $0.34-0.44$ & $2.4 \pm 0.9$ & $0.23-0.29$ & $2.9 \pm 0.7$ \\
\hline
\end{tabular}

Table 1: Perturbative thermal diffusivity measured with modulated ECH heat pulses and partial sawtooth crash-generated heat pulses. Modulated ECH data is taken from Reference [25].

against modulated ECH-based measurements, these new measurements will be utilized in order to examine both experimental trends of perturbative diffusivity and to validate gyrokinetic simulations. The discharges in the remainder of this paper highlight the advantage of the partial sawtooth measurement method, as none of them had modulated ECH data, and so one would not have been able to measure the perturbative diffusivity without the new method.

\section{Dependence of Perturbative Diffusivity on Collisionality}

As an initial application of the partial sawtooth crash-generated heat pulse measurement technique on ASDEX Upgrade, the perturbative thermal diffusivity was measured in 24 plasma discharges. These discharges were all L-mode plasmas, but had no particular constraints on any plasma parameters.

While the primary intent of analyzing these discharges was to confirm that the analysis technique could be successfully applied to a variety of cases, they also provided an opportunity to expand upon the parametric dependency analysis presented in Reference [5].

Reference [5] found that empirically, $\chi_{e}^{\text {pert }}$ depended most strongly on plasma density and temperature, but certainly did not exhaust every possible comparison. The combination of data from both Alcator CMod and ASDEX Upgrade opens up many additional dependency comparisons, as the two machines together can cover a much larger range of many plasma parameters than each machine individually.

All of the dependencies discussed in Reference 5] (temperature, density, gradients, etc.) were also calculated for the set of data from ASDEX Upgrade, and in all cases the trends between perturbative thermal diffusivity and the various parameters were similar on both machines. In addition, the availability of data from two fairly different machines (a factor of roughly 2.5 in size and 3 in magnetic field) allowed for the exploration of new parametric dependencies over a wider range, such as various normalizations of the
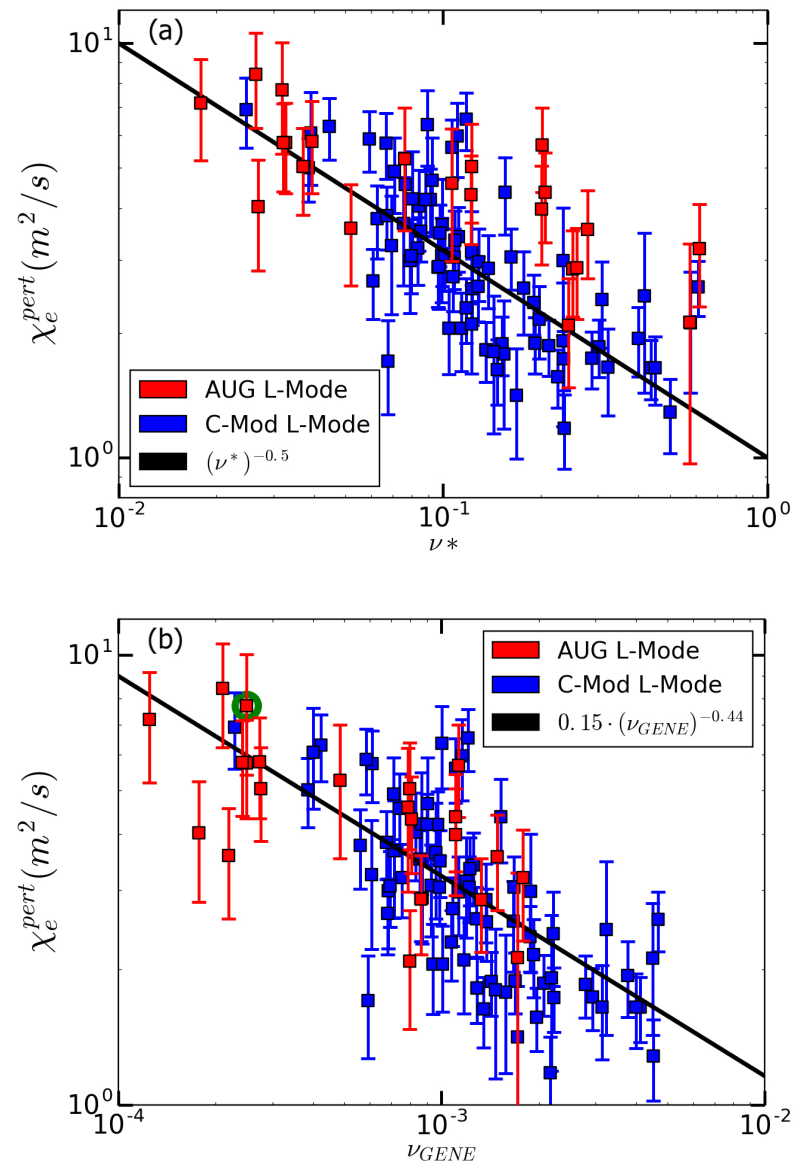

Figure 2: Log-log plots of the perturbative thermal diffusivity from 24 ASDEX Upgrade discharges (red) and 56 Alcator C-Mod discharges (blue) against two definitions of collisionality. All discharges are L-mode plasmas. (a) shows collision frequency normalized by the bounce frequency, and (b) shows a normalized gyrokinetic collisionality from GENE [28. Trend lines are shown in black. The point circled in green in (b) is the discharge considered in the simulations in Section 4. 
collision frequency. The data shown here consists of all 24 ASDEX Upgrade L-mode discharges, as well as the 56 L-mode discharges from Alcator C-Mod shown in Reference 5. Two normalizations of the collision frequency in particular will be highlighted here.

First, consider the following definition of collisionality, $\nu^{*}$, which is essentially the collision frequency normalized to the bounce frequency [29]:

$\nu^{*} \approx 0.01\left(\frac{R_{0}}{r}\right)^{3 / 2}\left(\frac{q R_{0} n_{20}}{T_{k}^{2}}\right)$

where $R_{0}$ is the major radius, $r$ is the local minor radius, $q$ is the safety factor, $n_{20}$ is the plasma density in units of $10^{20} \mathrm{~m}^{-3}$ and $T_{k}$ is the plasma temperature in units of $\mathrm{keV}$.

As is seen in Figure 2 (a), this particular definition of collisionality tends to bring the data from both of the machines into the same range. The figure also shows that the data overlay quite nicely, with increasing $\nu^{*}$ generally leading to decreasing perturbative diffusivity. This trend is consistent with the trends with temperature and density found in Alcator C-Mod, for which increasing density and decreasing temperature tended to lead to decreased perturbative diffusivity [5]. More quantitatively, a fit of $\left(\nu^{*}\right)^{-0.5}$ has an unweighted $R^{2}$ value of 0.53 , where $R^{2}$ quantifies how much of the variation in the perturbative diffusivity can be explained by variation in the parameter of interest. This exceeds all of the $R^{2}$ values given in Reference [5], despite including data from two different machines.

Consider next an alternative definition of the collisionality, for example the species-independent normalized collisionality used in the gyrokinetic code GENE [28]:

$\nu_{G E N E}=2.3031 \cdot 10^{-5} \frac{a \cdot n_{19} \cdot \ln \Lambda}{T_{k}^{2}}$

where $a$ is the square root of the edge toroidal flux divided by $\pi$ times the reference toroidal magnetic field, $a=\sqrt{\Phi_{\text {edge }} / \pi B_{\text {ref }}}$ (generally equal to the machine minor radius) [30, $n_{19}$ is the plasma density in units of $10^{19} \mathrm{~m}^{-3}, T_{k}$ is the plasma temperature in units of $\mathrm{keV}$, and $\ln \Lambda$ is the Coulomb Logarithm. Internally in GENE this quantity is labelled $\nu_{c}$. Note that GENE uses species dependent collision frequencies when performing the relevant calculations, but that this species-independent normalized collisionality can be used to parametrize the plasma in question 28.

In contrast to the previous definition of collisionality, this definition tends to separate the data from Alcator C-Mod and ASDEX Upgrade slightly more, leaving some regions on both extremes covered only by one machine. Figure 2 (b) shows the trend between $\nu_{G E N E}$ and the perturbative diffusivity. As with $\nu^{*}$, the perturbative diffusivity tends to decrease with increasing $\nu_{G E N E}$, though the exponent is slightly different $(-0.44$ instead of -0.5$)$. There is also a multiplicative factor of approximately 0.15 in front of the collisionality. This fit seems to describe the data almost equally well, with an $R^{2}$ value of 0.52 , which is almost identical to the value for $\nu^{*}$. In addition, the circled point in Figure 2 is the discharge on which the gyrokinetic simulations described in Section 4 are based.

The physical mechanisms behind the correlation between the perturbative thermal diffusivity and the collisionality are likely complex, but may be related to the relative strength of ITG (ion temperature gradient) and TEM (trapped electron mode) turbulence in these plasmas. As noted by Reference 31, lower collisionality generally favors stronger destabilization of TEM compared to ITG, while higher collisionality tends to increase the relative strength of ITG modes. Reference 32 shows that increased TEM drive may also correlate with increased perturbative diffusivity (as measured with modulated ECH). The new experimental results presented here from both Alcator C-Mod and ASDEX Upgrade are consistent with this interpretation.

As shown in this section, the physics of the perturbative thermal diffusivity are not machine dependent. The large number of discharges included here also reveals that this measurement method can reliably be applied to many different plasma conditions, and that such a measurement is ready for comparison to the outputs of gyrokinetic simulations, which will be presented in Section 4. The next section will also compare these experimentally observed trends to those predicted by gyrokinetic simulation.

\section{Validation of Local, Ion-Scale, Nonlinear GENE}

The perturbative thermal diffusivity will now be used as part of a validation study of the gyrokinetic code GENE on ASDEX Upgrade. Local, ion-scale $\left(k_{y} \rho_{s}<\right.$ 2.3), nonlinear GENE simulations of ASDEX Upgrade discharge \#33585 were performed at a radial location of $\rho_{\text {tor }}=0.49$ as part of this validation study, where $\rho_{\text {tor }}$ is the square root of the normalized toroidal flux. The location was chosen to be in the center of the radial region over which the perturbative thermal diffusivity of this discharge was measured $\left(\rho_{t o r}=0.44\right.$ - 0.54). These simulations included electromagnetic effects, used a realistic electron to ion mass ratio, included the effects of impurities through an effective charge $Z_{\text {eff }}$ (justified by comparing results of linear simulations with a third kinetic impurity species, tungsten, to those using $Z_{e f f}$, finding good agreement in the range of wavenumbers included in the nonlinear 
simulations), and treated collisions with a linearized Landau-Boltzmann operator. The simulations used the experimental magnetic equilibrium, as reconstructed with the CLISTE code 33.

Note that the validation study presented in this work is for the same plasma discharge used in the validation study in Reference 34. The work here, however, focuses on a different radial location and utilizes the perturbative thermal diffusivity as an additional validation constraint (for the first time on ASDEX Upgrade), instead of fluctuation measurements. Other than the radial location (and the accompanying changes in plasma parameters and shape), most other GENE settings were the same in both validation studies.

The particular discharge under consideration was an electron-heated, L-mode plasma on ASDEX Upgrade with toroidal field on axis $B_{t}=2.5 \mathrm{~T}$, plasma current $I_{p}=1.0 \mathrm{MA}$, and central density $n_{e, o}=2.6 \cdot 10^{19} \mathrm{~m}^{-3}$. This discharge was heated with $0.7 M W$ of electron cyclotron heating (ECH), deposited at $\rho_{\text {tor }} \approx 0.2$.

The electron temperature in this discharge was measured with an electron cyclotron emission (ECE) radiometer [14, the electron density with Thomson Scattering [35, 36], and the ion temperature and toroidal plasma rotation with charge exchange recombination spectroscopy (CXRS) based on periodic neutral beam blips [37. Please see Reference 34 for the full profiles in this discharge. These diagnostics have the following uncertainties, calculated by combining variations in time with the instrumental uncertainty quoted in each diagnostic reference: $15 \%$ in $T_{e}, 20 \%$ in $T_{i}, 10 \%$ in $n_{e}$, and $50 \%$ in $v_{r o t}$. Fitted profiles are utilized to calculate the normalized gradient scale lengths $a / L_{y}=-(a / y)\left(d y / d \rho_{t o r}\right)$, where $a$ is the square root of the edge toroidal flux divided by $\pi$ times the reference toroidal magnetic field, $a=\sqrt{\Phi_{\text {edge }} / \pi B_{\text {ref }}}$ (equal to the plasma minor radius), and $\mathrm{y}$ is the plasma parameter of interest 28. These have the following uncertainties, calculated by combining time uncertainties with uncertainties inferred from varying profile fits within diagnostic uncertainty: $20 \%$ in $a / L_{T e}, 30 \%$ in $a / L_{T i}$, and $30 \%$ in $a / L_{n e}$. The normalized gradient scale lengths are used as inputs to the GENE simulations, and will be varied within experimental uncertainty during sensitivity scans.

Table 2 gives the nominal experimental input parameters to the GENE simulations. The table also shows the normalized temperature gradient scale lengths $\left(a / L_{T_{i}}\right.$ and $\left.a / L_{T_{e}}\right)$ for the heat flux matched case, which will be discussed further in Section 4.2 .

The remainder of this section will discuss both linear and nonlinear results of these GENE simulations.
Table 2: Input parameters to GENE simulations in this validation study. The magnetic shear is $\hat{s}$ and the $E \times B$ shearing rate is $s_{E \times B}$. All values are the nominal experimental values, except for the values of the normalized temperature gradient scale lengths in parentheses, which are for the heat flux matched case discussed in Section $4.2\left(a / L_{T_{i}}\right.$ down by $19 \%$ and $a / L_{T_{e}}$ down by $\left.12 \%\right)$.

\begin{tabular}{cc}
\hline Quantity & Value \\
\hline$\rho_{\text {tor }}$ & 0.49 \\
$q$ & 1.42 \\
$\hat{s}$ & 0.728 \\
$s_{E \times B}\left[a / c_{s}\right]$ & 0.0042 \\
$a / L_{T_{i}}$ & $1.59(1.29)$ \\
$a / L_{T_{e}}$ & $3.27(2.88)$ \\
$a / L_{n}$ & 0.926 \\
$T_{e}[\mathrm{keV}]$ & 1.57 \\
$n_{e}\left[10^{19} \mathrm{~m}^{-3}\right]$ & 2.14 \\
$T_{i} / T_{e}$ & 0.41 \\
$\mathrm{Z}_{\text {eff }}$ & 1.6 \\
$\beta_{e}[\%]$ & 1.96 \\
$\nu_{c}$ & 0.000209 \\
$\nu_{\text {ei }}\left[a / c_{s}\right]$ & 0.081 \\
$R_{\text {axis }}[\mathrm{m}]$ & 1.65 \\
$r_{\text {minor }}[\mathrm{m}]$ & 0.325 \\
$B_{\text {ref }}=B_{0}[\mathrm{~T}]$ & 2.626 \\
$L_{\text {ref }}=a[\mathrm{~m}]$ & 0.652 \\
$c_{s}[\mathrm{~km} / \mathrm{s}]$ & 274.16 \\
\hline
\end{tabular}

First, the linear growth rates and real frequencies will be presented. Next, the results of sensitivity scans of $a / L_{T_{i}}$ and $a / L_{T_{e}}$ within experimental uncertainty will be presented, with the goal of matching the experimentally inferred electron and ion heat fluxes within uncertainty. These scans reveal that one must scan both $a / L_{T_{i}}$ and $a / L_{T_{e}}$ simultaneously in order to match the experimental heat fluxes, and also reveals interesting nonlinear dependencies of the simulated heat fluxes. Finally, the heat flux matched simulations will be compared to the additional validation constraint of the perturbative thermal diffusivity, finding for the first time good agreement between ion-scale gyrokinetic simulation and experimental perturbative diffusivity measured with partial sawteeth.

\subsection{Linear Results}

The results of linear GENE simulations for the plasma discharge considered in this study are shown in Figure 3. Figure 3 (a) shows the results for the nominal experimental gradients and (b) shows the results for the flux-matched case $\left(a / L_{T i}\right.$ reduced by $19 \%$ and $a / L_{T e}$ reduced by $\left.12 \%\right)$. In this figure, blue 
points represent modes with a positive real frequency, indicative of modes moving in the ion drift direction. Similarly, red points represent modes with a negative real frequency, indicative of modes moving in the electron drift direction. Diamonds represent the dominant mode at a given wavenumber, and circles represent subdominant modes.

Perhaps the most interesting observation from Figure 3 (a) is that in the ion-scale range of $k_{y} \rho_{s} \lesssim 2.0$, ion- and electron-modes alternate as the dominantly growing mode. The two modes are close throughout this region, and especially close when the ion mode

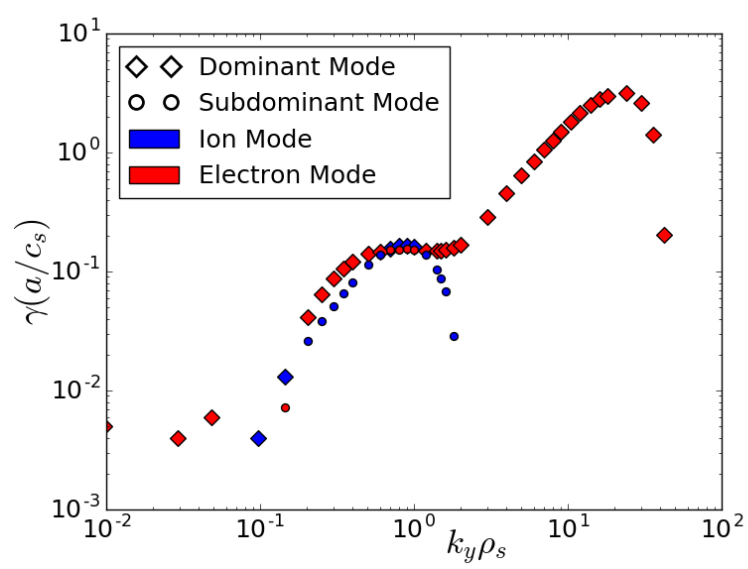

(a)

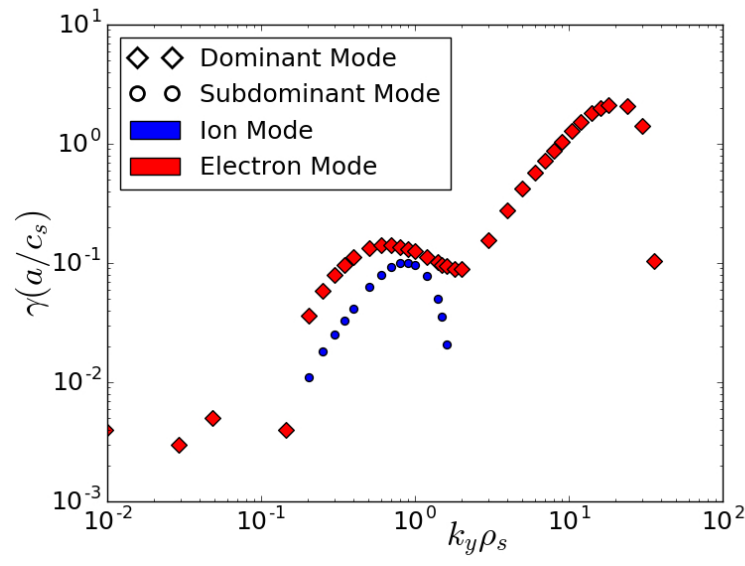

(b)

Figure 3: Linear stability results for discharge \#33585. Blue points have positive real frequency (the ion diamagnetic direction) and red points have negative real frequency (the electron diamagnetic direction). (a) shows the linear growth rates of the dominant and secondary modes for the nominal experimental parameters and (b) shows the results for the heat flux matched case $\left(a / L_{T i}\right.$ reduced by $19 \%$ and $a / L_{T e}$ reduced by $12 \%)$. becomes slightly dominant around $k_{y} \rho_{s} \approx 0.9$. This mixed-mode linear stability foreshadows the perhaps non-intuitive dependencies of the electron and ion heat fluxes on the input electron and ion temperature gradients which will be described further in Section 4.2 .

Such mixing of dominant modes also becomes clear when one compares linear stability results from the nominal experimental parameters and scanned input temperature gradients. With $a / L_{T i}$ reduced by $19 \%$ and input $a / L_{T e}$ reduced by $12 \%$ (the 'heat flux matched' case presented below), for example, the plots are similar in shape, but all of the dominant modes are electron modes, and there are no dominant ion modes, as shown in Figure 3 (b). The ion modes do nearly become dominant at $k_{y} \rho_{s} \approx 1.0$, indicating that even though the plasma is electronmode dominant according to linear stability, the plasma is still subdominantly mixed-mode.

\subsection{Nonlinear Heat Flux Comparison}

This section will explore the sensitivity of the nonlinear GENE heat fluxes to the input normalized temperature gradient scale lengths, and will compare the simulation and experimental heat fluxes. From a validation standpoint, matching the experimental heat fluxes to within uncertainty is a key component of confirming that GENE is correctly reproducing the real plasma behavior. In addition, exploring the sensitivity of the heat fluxes to the temperature gradients provides insight into the more fundamental nature of the turbulence in this particular plasma discharge.

These nonlinear simulations had two gyrokinetic species (the effects of impurities were included through an effective charge $Z_{e f f}$ ), 128 grid points in the radial direction, 48 bi-normal modes (corresponding to 96 grid points due to the hermiticity of the Fourier coefficients for real quantities [30]), 24 grid points in the parallel direction, 48 grid points in parallel velocity, and 16 points in magnetic moment (a resolution of $128 \times 96 \times 24 \times 48 \times 16$ grid points). $k_{y} \rho_{s}$ ranged from 0.048 to 2.3. This corresponds to a minimum finite toroidal mode number of 5 . The box extended $113.5 \rho_{s}$ in the radial direction, up to $3 v_{t h, j}$ in the parallel velocity (where $v_{t h, j}=\sqrt{2 T_{0 j} / m_{j}}$ ), and up to $9 T_{0 j} / B_{\text {ref }}$ in the magnetic moment. Convergence checks were performed around the resolution and box size (except in bi-normal modes, as this would begin to extend into the electron scale) to ensure that these values were all sufficient.

As stated above, the uncertainty in the normalized ion temperature gradient scale length, $a / L_{T i}$, is approximately $30 \%$, and the uncertainty in the normalized electron temperature gradient scale length, $a / L_{T e}$, is approximately $20 \%$. The GENE inputs are only varied within these bounds in order to be 
consistent with the experiment.

The experimental heat fluxes are calculated with the power balance transport solver TRANSP [38, giving $P_{e}=0.67 \mathrm{MW}$ and $P_{i}=0.21 \mathrm{MW}$ at this radius. Note that in this paper $P_{j}$ refers to a total power in MW while $Q_{j}$ refers to a heat flux in $\mathrm{MW} / \mathrm{m}^{2}$ $\left(P_{j}=S \cdot Q_{j}\right.$, where $S$ is the flux surface area). The uncertainties in the input parameters are propagated through the governing equations of TRANSP in order to find the uncertainties in the experimental heat fluxes. These turn out to be $18 \%$ for $P_{e}$ and $26 \%$ for $P_{i}$ 困

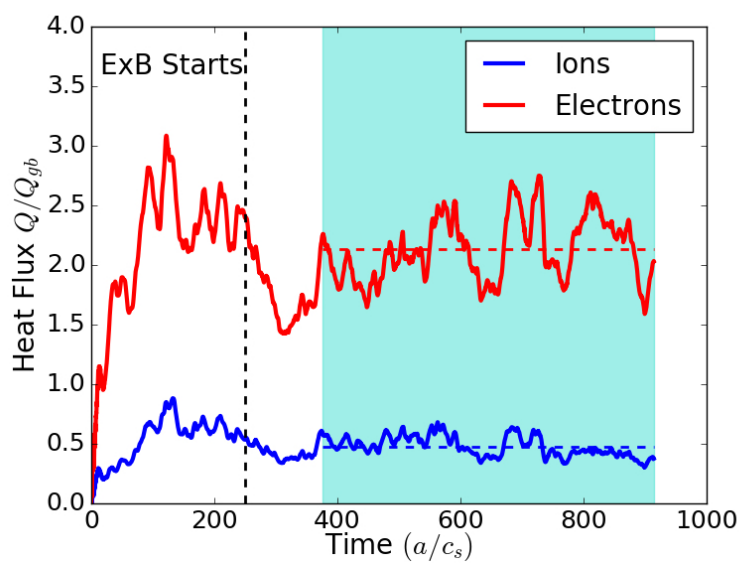

Figure 4: GENE heat flux output for nonlinear simulation of ASDEX Upgrade discharge \#33585 at $\rho_{\text {tor }}=0.49$. This is the 'heat flux matched' case described below, with input $a / L_{T i}$ reduced by $19 \%$ and input $a / L_{T e}$ reduced by $12 \%$. Plotted as Gyro-Bohm heat flux (electrostatic plus electromagnetic) against time in units of $a / c_{s}$. Electrons in red and ions in blue. Averaging performed over the time region highlighted in turquoise, with the dashed lines representing the average heat flux.

The GENE heat fluxes are calculated by averaging the total heat flux output (electrostatic and electromagnetic) from the simulation over several hundred $a / c_{s}$ times, where $c_{s}$ is the sound speed. Since the simulation outputs the heat flux several times during each $a / c_{s}$ time, these averages contain several thousand data points. The period of the time average only begins after initial transients have died off in the simulation, and the effect of $E \times B$ shear has been fully realized.

Figure 4 shows the time trace from the heat flux

$\ddagger$ Note that the uncertainty in $P_{e}$ quoted in this study $(18 \%)$ is larger than that in Reference 34] (14\%). This is due to additional uncertainty discovered in the routines that calculate the radiated power profiles since the publication of that study. This increase in uncertainty only strengthens the statements made in Reference 34. matched GENE run. The turquoise region highlights the period over which the simulation results are averaged, starting at $t=375 a / c_{s}$ and continuing for an additional $540 a / c_{s}$. The effects of $E \times B$ shear were initialized at $t=250 a / c_{s}$, and have therefore had time to come to steady state before the time averaging begins. The horizontal dashed lines represent the calculated average heat fluxes. The uncertainty on the heat flux is then calculated using the method described in Reference [39]. The particular case shown in Figure 4 gives $Q_{e}=(2.13 \pm 0.06) Q_{G B}$ and $Q_{i}=(0.48 \pm$ $0.04) Q_{G B}$. These values are then converted to units of MW by multiplying by the Gyro-Bohm heat flux, $Q_{G B}$, and the flux surface area, giving $P_{e}=0.73 \mathrm{MW}$ and $P_{i}=0.16 \mathrm{MW}$. The values produced by this averaging procedure were also used to check that the simulations had converged numerically, showing that increasing the box size or grid resolution did not change the results outside of uncertainty.

Even though this particular simulation shows a strong transient after the initialization of $E \times B$ shear, the average quickly returns to a value that is very similar to the heat flux before the $E \times B$ shear is turned on. This observation is common to all of the simulations considered in this study, and is consistent with the fairly small value of the $E \times B$ shearing rate, $s_{E \times B}$, that is input into these simulations. This is a result of the fairly flat rotation profile of the plasma at this radius.

Note that even without performing multi-scale simulations, it is possible to separately perform ionscale and electron-scale simulations, calculate heat fluxes, and linearly add these results together, though past work has shown that this procedure often gives poor agreement with real multi-scale simulations [6, 12. One might expect the electron-scale simulations to in some cases contribute to the electron heat flux in a meaningful way, but in the simulations considered here the very low ratio of the peak growth rate at electron scales to the peak growth rate at ion scales from Figure 3 (discussed later in further detail), would indicate that the electron scale contribution to the heat flux will be much smaller than the ion-scale contribution. For these reasons, only ion-scale simulations are performed as part of this work.

Sensitivity scans with GENE were performed by scanning the input ion and electron temperature gradients within experimental uncertainty. A number of interesting observations resulted from these scans. First, nonlinear GENE heat fluxes were found to agree with experimental heat fluxes within uncertainty when $a / L_{T i}$ was decreased by $19 \%$ and $a / L_{T e}$ was decreased by $12 \%$ (the case shown in Figure 4). These gradient scale length inputs result in predictions of $P_{e}=0.73 \mathrm{MW}$ and $P_{i}=0.16 \mathrm{MW}$, which are within 
Table 3: Summary of nonlinear GENE runs in this study. Input normalized gradient scale lengths, the simulation time over which the heat flux was averaged, and the output ion and electron heat fluxes are given. Rows are in order of decreasing ion temperature gradient and then decreasing electron temperature gradient. In Gyro-Bohm units, the experimental heat fluxes were $Q_{e}^{e x p}=1.97 \pm 0.35 Q_{G B}$ and $Q_{i}^{\text {exp }}=0.62 \pm 0.16 Q_{G B}$ where $Q_{G B}=0.016$ $\mathrm{MW} / \mathrm{m}^{2}$. The heat flux matched simulation is highlighted in green.

\begin{tabular}{|c|c|c|c|c|}
\hline$a / L_{T_{i}}$ & $a / L_{T_{e}}$ & $\begin{array}{c}\text { Averaged } \\
a / c_{s}\end{array}$ & $Q_{i}\left(Q_{G B}\right)$ & $Q_{e}\left(Q_{G B}\right)$ \\
\hline Nominal & Nominal & 110 & $2.50 \pm 0.15$ & $5.03 \pm 0.22$ \\
\hline$-10 \%$ & Nominal & 179 & $1.08 \pm 0.08$ & $4.41 \pm 0.29$ \\
\hline$-19 \%$ & $-12 \%$ & 540 & $0.48 \pm 0.04$ & $2.13 \pm 0.06$ \\
\hline$-20 \%$ & Nominal & 180 & $0.93 \pm 0.06$ & $7.12 \pm 0.55$ \\
\hline$-20 \%$ & $-13 \%$ & 370 & $0.41 \pm 0.05$ & $2.01 \pm 0.24$ \\
\hline$-20 \%$ & $-15 \%$ & 595 & $0.56 \pm 0.02$ & $1.42 \pm 0.08$ \\
\hline$-20 \%$ & $-20 \%$ & 300 & $1.02 \pm 0.03$ & $1.47 \pm 0.03$ \\
\hline$-21 \%$ & $-15 \%$ & 375 & $0.43 \pm 0.03$ & $1.53 \pm 0.07$ \\
\hline$-22 \%$ & $-15 \%$ & 290 & $0.34 \pm 0.01$ & $2.08 \pm 0.05$ \\
\hline$-24 \%$ & $-15 \%$ & 195 & $0.43 \pm 0.02$ & $3.03 \pm 0.11$ \\
\hline$-25 \%$ & $-15 \%$ & 300 & $0.37 \pm 0.03$ & $2.63 \pm 0.24$ \\
\hline
\end{tabular}

the the uncertainty of the TRANSP outputs listed above $\left(P_{e}^{e x p}=0.67 \pm 0.12 \mathrm{MW}\right.$ and $P_{i}^{e x p}=0.21 \pm 0.06$ $\mathrm{MW})$. This simulation will henceforth be referred to as the 'heat flux matched' simulation, since for the purposes of validation, this was the simulation that agreed best with experimental measurements. This is also the simulation that will be used for comparison with the perturbative thermal diffusivity in Section 4.3 , as validation requires all constraints to be met simultaneously.

Another result of these scans is that one must change both $a / L_{T i}$ and $a / L_{T e}$ simultaneously in order to match the electron and ion heat fluxes. In addition, one must scan both in order to match just the ion heat flux, consistent with the mixed mode nature of the plasma seen in the linear stability analysis. As shown in Table 3, the nominal experimental gradients overpredict $Q_{i}$ by roughly a factor of 4 and $Q_{e}$ by roughly a factor of 2.5 . Reducing $a / L_{T i}$ by $10 \%$ improves this to a factor of 2 overprediction of $Q_{i}$, and a factor of 2 overprediction of $Q_{e}$. Further reducing $a / L_{T i}$ to $20 \%$ below the nominal value has little effect on the predicted ion heat flux, but increases the predicted electron heat flux to more than a factor of 3 overprediction. This scan would seem to imply that one must also change $a / L_{T e}$ in order to simultaneously match both $Q_{i}$ and $Q_{e}$.

A scan of $a / L_{T e}$ (from nominal to $20 \%$ below

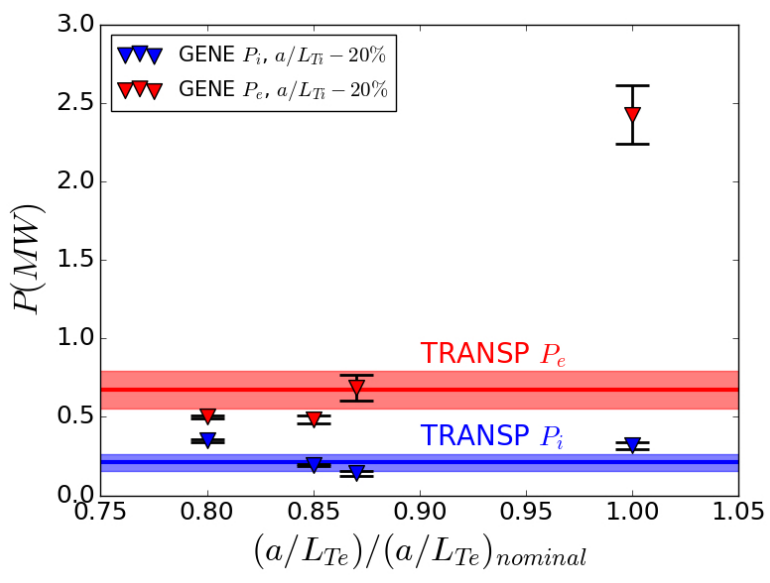

Figure 5: Comparison of experimental and simulation electron and ion heat fluxes at the same input ion temperature gradient and different input electron temperature gradients. Ion heat fluxes are given in blue and electrons in red. Experimental values are given by the solid lines, with uncertainty represented by the shaded regions. Simulation values are given by the triangles, with uncertainty represented with error bars.

nominal) at a fixed $a / L_{T i}$ reduction of $20 \%$ below the nominal value is shown in Figure 5. Electrons are shown in red and ions in blue. Uncertainty is represented by the black error bars. Reducing $a / L_{T e}$ from the nominal value at fixed $a / L_{T i}$ initially reduces $P_{e}$ from a large overprediction to roughly the experimental level of heat flux (at $\left.-13 \% a / L_{T e}\right)$, agreeing within uncertainty. Further reduction then brings the predicted $P_{e}$ to below the experimental uncertainty. Interestingly, at this fixed value of $a / L_{T i}$, changing $a / L_{T e}$ leads to a non-monotonic behavior of $P_{i}$. Reducing $a / L_{T e}$ by $13 \%$ reduces the predicted $P_{i}$ from slightly less than a factor of 2 overprediction to just below the experimental uncertainty in the measured $P_{i}$ (though this is difficult to see in the figure since the symbol has finite size). It turns out that if one considers both the experimental uncertainty and the simulation uncertainty, this simulation marginally agrees with the experiment, though not nearly as well as the 'heat flux matched' simulation described above. Further reduction of $a / L_{T e}$, however, actually begins to increase the predicted $P_{i}$, back to a level larger than the experimental value.

The result of instead scanning $a / L_{T i}$ at fixed $a / L_{T e}$ is shown in Figure 6. Note that Figures 5 and 6 have different $\mathrm{x}$ - and $\mathrm{y}$-axes. In this figure, the circles represent a scan of $a / L_{T i}$ between $25 \%$ and $20 \%$ below the nominal value, all at a fixed $a / L_{T e}$ reduction of $15 \%$. While the non-monotonic behavior is weaker than when scanning $a / L_{T e}$ at 


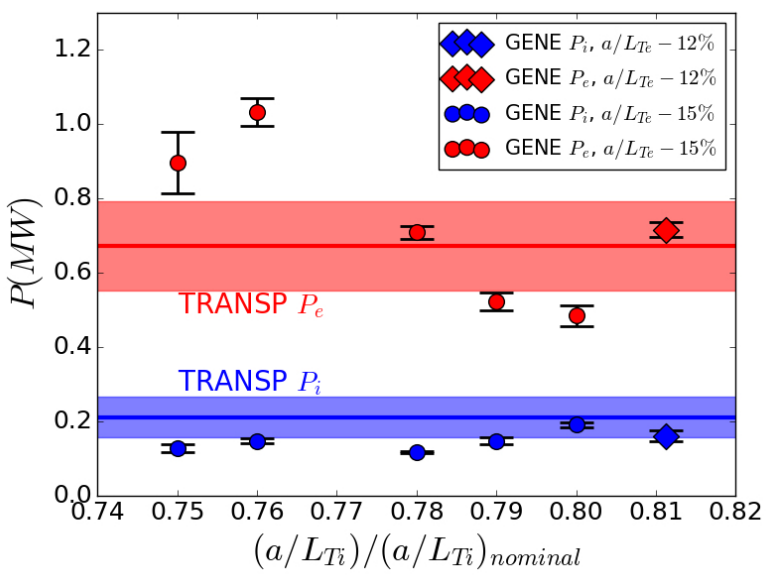

Figure 6: Comparison of experimental and simulation electron and ion heat fluxes at different input ion temperature gradients. Ion heat fluxes are given in blue and electrons in red. Experimental values are given by the solid lines, with uncertainty represented by the shaded regions. Simulation values at $a / L_{T e}-15 \%$ are given by the circles, with uncertainty represented with error bars. The flux matched case, with $a / L_{T e}-12 \%$ and $a / L_{T e}-19 \%$, is shown as the diamonds.

fixed $a / L_{T i}$, it does also occur outside of simulation uncertainty. The point at $-20 \% a / L_{T i}$ and $-15 \% a / L_{T e}$ is from the same simulation on both plots. This figure also includes the data from the heat flux matched simulation, represented as the diamonds.

In practice, the heat flux matched simulation inputs $\left(a / L_{T i}\right.$ reduced by $19 \%$ and $a / L_{T e}$ reduced by $12 \%)$ were obtained iteratively, resulting in the scans presented in both Figures 5 and 6. $a / L_{T i}$ was first reduced to get closer to the experimental values of $Q_{i}$ and $Q_{e}$, and then $a / L_{T e}$ was scanned at fixed values of $a / L_{T i}$. Due to the highly nonlinear dependencies of the heat fluxes, this process is not particularly straightforward, and may require many simulations. Table 3 shows some, but not all, of the simulations performed as part of this iterative process, excluding those simulations that were ended after early results revealed that a heat flux match was unlikely.

One may note that a few simulations on both of these plots agree well with $P_{i}$, but somewhat underpredict $P_{e}$. As stated above, it is possible that electron scales contribute some small portion to the electron heat flux, though the small growth rate at these scales suggests that this contribution would be small. It is therefore possible that some of these simulations would also agree with experiment to within uncertainty. It is unlikely, however, that they would agree better than the flux-matched simulation, and as stated before, linearly adding heat fluxes from different scales is not particularly rigorous.
The non-monotonic behavior of $Q_{i}$ with regards to both $a / L_{T i}$ and $a / L_{T e}$ is consistent with the results of linear stability analysis, suggesting that the plasma under consideration in this study is highly mixed-mode at the ion scales.

\subsection{Nonlinear Perturbative Diffusivity Comparison}

In addition to the electron and ion heat fluxes, the perturbative thermal diffusivity, as measured with the method described in Section 2, will be applied as a validation constraint to these GENE simulations. The discharge that was used for the GENE simulations presented here was one of the discharges described in Section 3, and is circled in Figure 2, Experimentally the plasma had a perturbative thermal diffusivity of $\chi_{E x p}^{\text {pert }}=7.7 \pm 2.3 \mathrm{~m}^{2} / \mathrm{s}$, averaged over the radial range of $\rho_{\text {tor }}=0.44-0.54$. This is the value that will be compared to the GENE outputs and it is for this reason that the GENE simulations were performed at $\rho_{\text {tor }}=0.49$.

As in previous work [5, 6, 7, 8, the simulation perturbative thermal diffusivity is calculated by taking the flux matched simulation from above, and scanning the input electron temperature gradient up and down by $6 \%$ and $12 \%$, which approximates the temperature gradient change induced by the passing of the partial sawtooth heat pulse. The electron heat flux output from the simulation is then plotted against the input gradient, and the slope of the curve at the flux matched simulation is used to calculate the perturbative thermal diffusivity. More quantitatively:

$\chi_{G E N E}^{p e r t}=\frac{a}{n_{e} T_{e}} \frac{\partial Q_{e}}{\partial\left(a / L_{T e}\right)}=\frac{1}{n_{e}} \frac{\partial Q_{e}}{\partial \nabla T_{e}}$

Note here that the turbulent time scales are much shorter than time scales of any macroscopic changes in the profiles. For example, the sawtooth period is approximately $25 \mathrm{~ms}$, compared to an $a / c_{s}$ time of approximately $2.4 \mu \mathrm{s}$. Even if turbulence saturates on a time scale of $100 a / c_{s}$, this leaves 100 saturation times in a single sawtooth period.

Figure 7 shows the results of this scan in $a / L_{T e}$ from the heat flux matched simulation, which is circled. In this figure, the heat flux is plotted against $n_{e} \nabla T_{e}$ so that the slope of the line has the correct units of perturbative thermal diffusivity. The experimental value of the perturbative diffusivity is plotted as the dash-dotted turquoise line going through the heat flux matched simulation, with the shaded region representing the experimental uncertainty.

The GENE simulations, shown as red squares, are fit with an exponential curve (red dotted line). The slope of the exponential fit at the heat flux matched simulation (red line) is $8.8 \mathrm{~m}^{2} / \mathrm{s}$. One would not expect the GENE simulations themselves to fall within the 


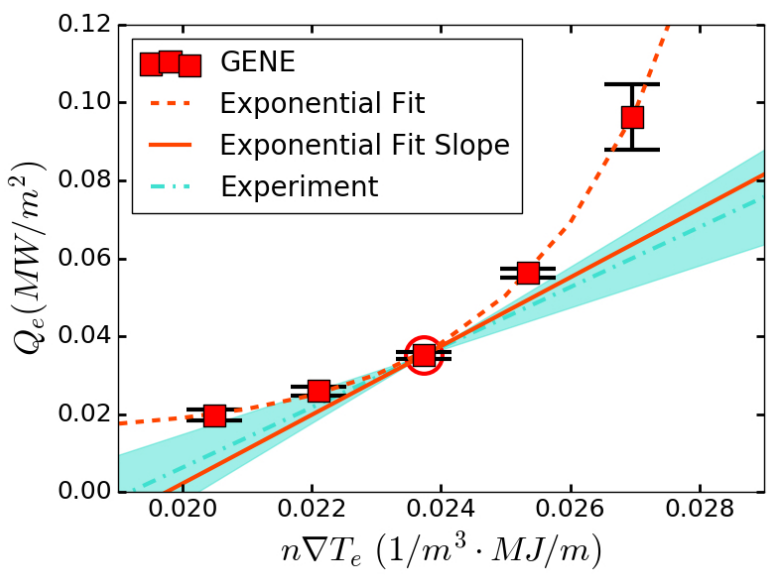

Figure 7: Perturbative thermal diffusivity measured experimentally and calculated from a scan of $a / L_{T e}$ in GENE simulations. GENE simulations are plotted as red squares with heat flux uncertainty represented as black error bars. The flux-matched simulation is circled in red. Perturbative diffusivity is a slope on this plot. The experimental perturbative diffusivity is represented as the dash-dotted turquoise line passing through the flux-matched simulation, with the shaded region representing the experimental uncertainty. An exponential fit to the GENE simulations is shown as the orange dashed line. The slope of this fit tangent to the flux-matched simulation is shown as the solid orange line. One should compare the turquoise (experiment) and orange (slope of exponential fit) lines in this figure.

shaded turquoise region (only the slope tangent to the fit) unless there was an exactly linear relationship between heat flux and temperature gradient. These results reveal that the slope of the exponential fit to the GENE simulations agrees with the experimental measurement to within uncertainty. Note that a linear fit to the GENE simulations (which is clearly inferior to the exponential fit) gives a perturbative diffusivity of $11.3 \mathrm{~m}^{2} / \mathrm{s}$, slightly outside of the experimental uncertainty.

This result is a stark contrast to past results of ion-scale simulations on Alcator C-Mod, such as those in References [5, 6, 7, 8, 12]. In past cases (with the gyrokinetic code GYRO [40] on Alcator CMod [41), ion-scale gyrokinetic simulations robustly underpredicted the perturbative thermal diffusivity, often by an order of magnitude. This was true even in cases where the simulations were able to simultaneously match the experimental ion and electron heat fluxes $[8$. Only the inclusion of multiscale effects was able to resolve this discrepancy [6, 8]. See Reference [42] for one recent verification study of GENE and GYRO, and References 43, 44, for an indirect benchmark, though in general, comparison of different gyrokinetic codes is beyond the scope of this work.

The value calculated from the exponential fit is much closer to the experimental value than in any of the previously reported ion-scale simulations, and actually slightly overpredicts the experimental value, also in contrast to previous results. This result strongly supports the theory that multi-scale effects and cross-scale coupling are very important to turbulent transport in some plasma discharges, but that ion-scale simulations are fully sufficient in other discharges. In particular, cases have now been identified in which: ion scale simulations miss both heat fluxes and perturbative diffusivity, requiring the inclusion of multi-scale effects [6]; ion scale simulations match heat fluxes, but miss the perturbative diffusivity 8; and ion scale simulations can simultaneously match both heat fluxes and perturbative diffusivity (the work presented here).

Another conclusion from these GENE simulations is that the manner in which the flux-gradient curve is fit can have an impact on the agreement with experiment. As mentioned above, the slope of the exponential fit agrees with experiment to within uncertainty, but the slope of a linear fit disagrees slightly outside of uncertainty. This is perhaps not surprising, as one might not expect an entirely linear response of the heat flux to the temperature gradient. It does, however, reveal that one should be careful when one fits these curves for comparison with experiment.

To put the strong agreement between experiment and simulation with electron and ion heat fluxes and perturbative diffusivity into context, consider the validation metric, $\chi$, originally developed in Reference [45] and used in Reference [34] (not to be confused with the perturbative diffusivity $\left.\chi_{e}^{\text {pert }}\right) . \quad \chi$ is to some extent a weighted sum of the differences between the experimental and simulated quantities. Lower $\chi$ represents better agreement between experiment and simulation, with $\chi<0.5$ being defined as generally agreeing, and $\chi>0.5$ being defined as generally disagreeing. Using $Q_{e}, Q_{i}$, and $\chi_{e}^{\text {pert }}$ (from the exponential fit) in this metric for the heat flux matched simulation gives $\chi=0.05$, indicating excellent agreement. This is better than any of the agreements found in Reference [34, though different constraints were used there. Note that this strong agreement is only possible after varying the input gradients within experimental uncertainty, as is common practice in turbulent transport validation studies [4].

A final application of these GENE simulations concerns the experimental trend of perturbative diffusivity with collisionality observed in Figure 2(b) in Section 3 . The experimental discharge that formed the 
basis for the GENE simulations in this section (AUG 33585) is circled in Figure 2 (top left of the plot). In order to see if GENE reproduces the same general trend of perturbative diffusivity with collisionality, the GENE collisionality $\left(\nu_{G E N E}\right.$ is called $\nu_{c}$ in the GENE code) was artificially raised by an order of magnitude from the flux matched simulation, with all other parameters left the same. The same was done for two of the simulations that scanned $a / L_{T e}$ in order to calculate the perturbative diffusivity $\left(a / L_{T e} 12 \%\right.$ above and below the flux matched simulation). These three new simulations were then used to calculate the perturbative diffusivity at the new collisionality.

This resulted in a perturbative diffusivity of 0.23 $\mathrm{m}^{2} / \mathrm{s}$, a decrease by a factor of 38 compared to the nominal collisionality for the flux matched case, which gave $8.8 \mathrm{~m}^{2} / \mathrm{s}$. Based on the experimental trend of $\nu_{G E N E}^{-0.44}$, one would expect the simulation to reduce by a factor of roughly 3 when the collisionality is increased by a factor of $10\left(10^{-0.44} \approx 0.36\right)$. The simulation therefore overestimates the dependence of perturbative thermal diffusivity on collisionality, predicting a much stronger decrease in perturbative diffusivity with increasing collisionality.

While it is in theory possible to scan the experimental collisionality while keeping all other dimensionless parameters constant, the authors would like to note that this particular parameter change contrasts starkly with all of the other input parameter adjustments performed in this study, which were performed strictly within experimental uncertainty. As such, the result presented here should be interpreted as a possible insight into the dependence on collisionality, instead of as a strict validation result. In the future, dedicated experimental collisionality scans will further investigate this dependence.

One interesting observation, however, is that the very small value predicted for the perturbative diffusivity, $0.23 \mathrm{~m}^{2} / \mathrm{s}$, is much closer to the values predicted by ion-scale gyrokinetic simulations of higher collisionality Alcator C-Mod plasmas in References [5, 6, 7, 8, In other words, when the collisionality of the discharge considered in this study is artificially increased to be of similar magnitude as that observed in Alcator C-Mod plasmas, a similar order of magnitude underprediction of the expected experimental perturbative diffusivity is observed. This result suggests that perhaps ion-scale simulations are better able to predict the perturbative diffusivity in low collisionality plasmas than in high collisionality plasmas. This may be related to the impact of the collisionality on the relative strength of ITG and TEM turbulence, as discussed above, but further investigation will be the topic of future work. Direct fluctuation measurements made during the propagation of partial sawtooth heat pulses may also shed light on this result, but would require further diagnostic development to obtain sufficient localization and time resolution.

\subsection{Interpretation of GENE Results}

The results from GENE presented in this study fit nicely into a larger story that has been built over the course of many validation studies in recent years. Various studies in recent years have made increasingly clear the importance of multi-scale effects and crossscale coupling in gyrokinetic simulations of some plasma discharges, with References [5, 6, 8, 12, 13, 43, 46, 47, 48, as just a few examples. Some of this work has indicated that comparing more than just experimental heat fluxes to the simulation results is necessary to fully differentiate multi- and ion-scale models. For example, in Reference [8], both ion-scale and multi-scale simulations are able to simultaneously match the electron and ion heat fluxes, albeit at slightly different input gradients, but only the multiscale simulation was able to correctly reproduce the perturbative diffusivity (after ion temperature stiffness effects were accounted for).

These and other references have suggested, however, that multi-scale effects may not always be important, and that in some plasmas ion-scale simulations may be perfectly sufficient to describe plasma transport. Practically, such a conclusion would mean that it is not always necessary to run computationally expensive multi-scale simulations in order to correctly simulate plasma behavior. The plasma simulated in this paper seems to be one such case.

The previous section commented on the apparent connection between the plasma collisionality and the necessity of multi-scale effects to correctly match the perturbative diffusivity, and the fact that this connection may be related to the relative strength of ITG and TEM turbulence at long wavelengths. In addition, past literature has proposed that one can distinguish plasmas in which multi-scale effects are important from those in which they are not, without actually running multi-scale simulations, by comparing the maximum growth rate from linear results at ionscales and electron-scales [12.

Specifically, one computes $\gamma_{h i g h-k} / \gamma_{l o w-k}$, where $\gamma_{h i g h-k}$ is the maximum linear growth rate of any mode above $k_{y} \rho_{s} \gtrsim 2.0$, and $\gamma_{l o w-k}$ is the maximum linear growth rate of any mode below $k_{y} \rho_{s} \lesssim 2.0$. Reference [12] suggests that plasmas in which $\gamma_{h i g h-k} / \gamma_{\text {low }-k} \lesssim$ 40 may not have significant multi-scale effects and that ion-scale simulations would be sufficient. Reference [8], however, presented a case in which $\gamma_{\text {high-k}} / \gamma_{\text {low }-k} \approx$ 30 and multi-scale interactions were required to match the perturbative diffusivity (but not the heat fluxes), 
so the value of $\gamma_{h i g h-k} / \gamma_{l o w-k}$ below which multi-scale effects are less important may be somewhat lower than previously estimated.

Based on the linear results for the discharge considered here, presented in Section 4.1. this ratio is $\gamma_{\text {high }-k} / \gamma_{\text {low }-k} \approx 19$ for the nominal experimental parameters and $\gamma_{h i g h-k} / \gamma_{\text {low }-k} \approx 15$ for the flux matched simulation. These values are both much lower than for the plasmas considered in References [8, 12, 13, and are consistent with the hypothesis that a lower value of $\gamma_{h i g h-k} / \gamma_{l o w-k}$ leads to decreased importance of multi-scale effects. Collisionality may also impact this ratio, combining several sets of observations in this work. The results presented here, combined with those from past work, suggest that the cutoff in $\gamma_{\text {high }-k} / \gamma_{\text {low }-k}$ may be somewhere in the range of 20 to 30 , as opposed to the value of 40 previously hypothesized.

While multi-scale GENE (and other gyrokinetic code) simulations would further strengthen the conclusions of this work, the enormous computational expense of such simulations are prohibitive, and are therefore beyond the scope of the current study. The results presented here are, however, excellent motivation for the eventual allocation of resources for multiscale GENE simulations.

\section{Conclusions}

This paper has presented the first measurements of the electron perturbative thermal diffusivity on ASDEX Upgrade using partial sawtooth-generated heat pulses. Calculating the perturbative diffusivity by averaging together the results of many individual pulses and by computing the perturbative diffusivity from a single 'composite' pulse were shown to agree, resolving one difference in the way that past literature calculated this quantity. The perturbative diffusivity measured with partial sawteeth was also compared to that measured with modulated electron cyclotron heating, which is a more established measurement method, finding good agreement (with the caveat that the radial ranges don't quite overlap). This method was applied to many ASDEX Upgrade discharges and combined with data from Alcator C-Mod, finding cross-machine trends between perturbative diffusivity and collisionality.

This partial sawtooth measurement technique was then utilized in a validation study of the gyrokinetic code GENE. An electron-heated L-mode plasma on ASDEX Upgrade was chosen, and simulated with local, non-linear, ion-scale simulations at $\rho_{\text {tor }}=0.49$. Linear analysis revealed that this plasma was strongly mixedmode in the ion-scales, with ion- and electron-modes alternating dominance for the nominal experimental parameters. Nonlinear simulations were able to simultaneously match both ion and electron heat fluxes within experimental uncertainty; though this required changing both $a / L_{T i}$ and $a / L_{T e}$ within uncertainty. This work is the first to show that an ion-scale gyrokinetic simulation is able to simultaneously match the perturbative thermal diffusivity, ion heat flux, and electron heat flux of an experimental plasma. Past ion-scale studies have shown robust underprediction of the perturbative diffusivity, motivating the use of multi-scale gyrokinetic simulations. The very low ratio of $\gamma_{h i g h-k} / \gamma_{\text {low }-k} \lesssim 20$ may be responsible for the lack of importance of multi-scale effects in this plasma and thus the good experimental agreement with an ion-scale simulation. The effect of increasing the simulation collisionality on the predicted perturbative diffusivity was also investigated. This work provides direct evidence that multi-scale simulations are not required to correctly simulate all plasmas, and that linear stability results may help differentiate between plasmas in which multi-scale effects are and are not important.

\section{Acknowledgements}

This work has been carried out within the framework of the EUROfusion Consortium and has received funding from the Euratom research and training programme 2014-2018 under grant agreement No 633053. The views and opinions expressed herein do not necessarily reflect those of the European Commission. This work is also supported by the US DOE under Grants DESC0006419, DE-FC02-99ER54512-CMOD, and DESC0017381. In addition, this research is supported by the US DoD and the Air Force Office of Scientific Research under the National Defense Science and Engineering Graduate (NDSEG) Fellowship, 32 CFR 168 a.

\section{References}

[1] AIAA 1998 Measurement of thermal transport by synchronous detection of modulated electron cyclotron heating in the doublet iii tokamak Tech. Rep. AIAA G-0771998 (2002) The American Institute of Aeronautics and Astronautics

[2] Jenko F, Dorland W and Kotschenreuther M 2000 Phys. Plasmas 71904

[3] Greenwald M 2010 Phys. Plasmas 17058101

[4] Holland C 2016 Phys. Plasmas 23060901

[5] Creely A J, White A E, Edlund E M, Howard N T and Hubbard A E 2016 Nucl. Fusion 56036003

[6] Howard N T, Holland C, White A E, Greenwald M and Candy J 2016 Nucl. Fusion 56014004

[7] Creely A J, Howard N T, Rodriguez-Fernandez P, NCao, Hubbard A E, Hughes J W, Rice J E, White A E, Candy J, Staebler G M, Conway G D, Freethy S J and Sung C 2017 Phys. Plasmas 24056104

[8] Howard N T, Holland C, White A E, Greenwald M, Rodriguez-Fernandez P, Candy J and Creely A J 2018 Plasma Phys. Controlled Fusion 60014034 
[9] Cardozo N J L 1995 Plasma Phys. Control. Fusion 37799

[10] Hogeweij G 2010 Fusion Sci. Technol. 57 357-363

[11] Citrin J, Jenko F, Mantica P, Told D, Bourdelle C, Garcia J, Haverkort J W, Hogeweij G M D, Johnson T and Pueschel M J 2013 Phys. Rev. Lett. 111155001

[12] Howard N T, Holland C, White A E, Greenwald M, Candy J and Creely A J 2016 Phys. Plasmas 23056109

[13] Holland C, Howard N and Grierson B 2017 Nucl. Fusion 57066043

[14] Willensdorfer M, Denk S S, Strumberger E, Suttrop W, Vanovac B, Brida D, Cavedon M, Classen I, Dunne M, Fietz S, Fischer R, Kirk A, Laggner F M, Liu Y Q, Odstrcil T, Ryan D A, Viezzer E, Zohm H, Luhmann I C, the ASDEX Upgrade Team and Team T E M 2016 Plasma Phys. Control. Fusion 58114004

[15] Fredrickson E D, Austin M E, Groebner R, Manickam J, Rice B, Schmidt G and Snider R 2000 Phys. Plasmas 7 5051

[16] DeLuca F, Galli P, Gorini G, Jacchia A, Mantica P, Deliyanakis N, Erba M and Porte L 1996 Nucl. Fusion 36909

[17] Tubbing B J D, Cardozo N L and der Wiel M V 1987 Nucl. Fusion 271843

[18] Cardozo N J L, Tubbing B, Tibone F and Taroni A 1988 Nucl. Fusion $\mathbf{2 8} 1173$

[19] Evensen H, Fonck R, Paul S and Scott S 1999 Nucl. Fusion 39133

[20] Ryter F, Leuterer F, Pereverzev G, Fahrbach H U, Stober J, Suttrop W and the ASDEX Upgrade Team 2001 Phys. Rev. Lett. 862325

[21] Ryter F, Tardini G, Luca F D, Fahrbach H U, Imbeaux F, Jacchia A, Kirov K, Leuterer F, Mantica P, Peeters A, Pereverzev G, Suttrop W and the ASDEX Upgrade Team 2003 Nucl. Fusion 431396

[22] Ryter F, Dux R, Mantica P and Tala T 2010 Plasma Phys. Control. Fusion 52124043

[23] Ryter F, Angioni C, Giroud C, Peeters A G, Biewer T, Bilato R, Joffrin E, Johnson T, Leggate H, Lerche E, Madison G, Mantica P, Eester D V, Voitsekhovitch I and Contributors J 2011 Nucl. Fusion 51113016

[24] DeBoo J C, Petty C C, White A E, Burrell K H, Doyle E J, Hillesheim J C, Holland C, McKee G R, Rhodes T L, Schmitz L, Smith S P, Wang G and Zeng L 2012 Phys. Plasmas 19082518

[25] Schneider P, Bustos A, Hennequin P, Ryter F, Bernert M, Cavedon M, Dunne M, Fischer R, Goerler T, Happel $\mathrm{T}$, Igochine $\mathrm{V}$, Kurzan B, Lebschy A, McDermott R, Morel P, Willensdorfer M, the ASDEX Upgrade Team and Team T E M 2017 Nucl. Fusion 57066003

[26] Jacchia A, Mantica P, Luca F D and Gorini G 1991 Phys. Fluids B 33033

[27] Mantica P, Luca F D, Gorini G, Jacchia A, Hogeweij G M D, Cardozo N J L, Kramer G J and Sips A C C 1992 Nucl. Fusion 322203

[28] Gene Development Team 2015 The Gyrokinetic Plasma Turbulence Code Gene: User Manual

[29] Freidberg J 2007 Plasma Physics and Fusion Energy (Cambridge University Press)

[30] Told D, Jenko F, Görler T, Casson F J, Fable E and the ASDEX Upgrade Team 2013 Phys. Plasmas 20122312

[31] Angioni C, Peeters A G, Jenko F and Dannert T 2005 Phys. Plasmas 12112310

[32] Ryter F, Angioni C, Peeters A G, Leuterer F, Fahrbach H U, Suttrop W and the ASDEX Upgrade Team 2005 Phys. Rev. Lett. 95085001

[33] McCarthy P J 1999 Phys. Plasmas 63554

[34] Freethy S J, Goerler T, Creely A J, Conway G D, Happel $\mathrm{T}$, Koenen C, Hennequin P, White A E and the ASDEX Upgrade Team 2018 Phys. Plasmas 25055903

[35] Murmann H, Goetsch S, Roehr H, Salzmann H and Steuer
K H 1992 Rev. Sci. Instrum. 634941

[36] Kurzan B and Murmann H D 2011 Rev. Sci. Instrum. 82 103501

[37] Viezzer E, Puetterich T, Dux R, McDermott R M and the ASDEX Upgrade Team 2012 Rev. Sci. Instrum. 83 103501

[38] Budny R, Bell M, Biglari H, Bitter M, Bush C, Cheng C, Fredrickson E, Grek B, Hill K, Hsuan H, Janos A, Jassby D, Johnson D, Johnson L, LeBlanc B, McCune D, Mikkelsen D, Park H, Ramsey A, Sabbagh S, Scott S, Schivell J, Strachan J, Stratton B, Synakowski E, Taylor G, Zarnstorff M and Zweben S 1992 Nucl. Fusion 32429

[39] Oberparleiter M, Nordman H, Verdoolaege G and Jenko F 2016 J. Phys.: Conf. Ser. $\mathbf{7 7 5} 012009$

[40] Candy J and Waltz R E 2003 J. Comput. Phys. 186 545581

[41] Greenwald M, Bader A, Baek S, Bakhtiari M, Barnard H, Beck W, Bergerson W, Bespamyatnov I, Bonoli P, Brower D, Brunner D, Burke W, Candy J, Churchill M, Cziegler I, Diallo A, Dominguez A, Duval B, Edlund E, Ennever P, Ernst D, Faust I, Fiore C, Fredian T, Garcia O, Gao C, Goetz J, Golfinopoulos T, Granetz R, Grulke O, Hartwig Z, Horne S, Howard N, Hubbard A E, Hughes J, Hutchinson I, Irby J, Izzo V, Kessel C, LaBombard B, Lau C, Li C, Lin Y, Lipschultz B, Loarte A, Marmar E, Mazurenko A, McCracken G, McDermott R, Meneghini O, Mikkelsen D, Mossessian D, Mumgaard R, Myra J, Nelson-Melby E, Ochoukov R, Olynyk G, Parker R, Pitcher S, Podpaly Y, Porkolab M, Reinke M, Rice J, Rowan W, Schmidt A, Scott S, Shiraiwa S, Sierchio J, Smick N, Snipes J A, Snyder P, Sorbom B, Stillerman J, Sung C, Takase Y, Tang V, Terry J, Terry D, Theiler C, Tronchin-James A, Tsujii N, Vieira R, Walk J, Wallace G, White A E, Whyte D G, Wilson J, Wolfe S, Wright G, Wright J, Wukitch S and Zweben S 2014 Phys. Plasmas 21110501

[42] Mikkelsen D R, a N T H, White A E and Creely A J 2018 Phys. Plasmas 25042505

[43] Görler T and Jenko F 2008 Phys. Rev. Lett. 100185002

[44] Candy J, Waltz R E, Fahey M R and Holland C 2007 Plasma Phys. Control. Fusion 491209

[45] Ricci P, Theiler C, Fasoli A, Furno I, Gustafson K, Iraji D and Loizu J 2011 Phys. Plasmas 18032109

[46] Howard N T, White A E, Reinke M L, Greenwald M, Holland C, Candy J and Walk J 2013 Nucl. Fusion 53 123011

[47] Howard N T, Holland C, White A E, Greenwald M and Candy J 2014 Phys. Plasmas 21112510

[48] Maeyama S, Idomura Y, Watanabe T H, Nakata M, Yagi M, Miyato N, Ishizawa A and Nunami M 2015 Phys. Rev. Lett. 114255002 